\title{
Cellular Automata Based Models of Wireless Sensor Networks
}

\author{
Wei Li \\ School of Information Technologies, \\ The University of Sydney \\ NSW 2006, Australia \\ liwei@it.usyd.edu.au
}

\author{
Albert Y. Zomaya \\ School of Information Technologies, \\ The University of Sydney \\ NSW 2006, Australia \\ a.zomaya@usyd.edu.au
}

\author{
Adel Al-Jumaily \\ School of Electrical, Mechanical \\ and Mechatronic Systems, Faculty \\ of Engineering and Information \\ Technology, University of Technology, \\ Sydney, NSW 2007, Australia
}

\begin{abstract}
Large scale wireless sensor networks present interesting challenges in many applications due to a number of factors, such as, energy constraints, the dynamic nature of the network topology and various application needs. An important issue is how the sensor nodes can achieve efficient global behavior under distributed control mechanisms? One of many possible solutions is to construct a self-organized wireless sensor network to deal with this challenge. This paper presents an algorithm to construct a self-organized wireless sensor network based on two dimensional cellular automata that can provide better understanding for how local behavior at node level influences the overall system behavior and affect the system performance. Two types of Cellular Automata (CA) are considered; for the synchronous CA based system; the regular patterns are identified and discussed. To overcome some limitations arising from the use of a synchronous CA implementation an asynchronous CA is employed.
\end{abstract}

\section{Categories and Subject Descriptors}

C.2.1 [Computer-Communication networks]: Network Architecture and Design - network topology

\section{General Terms}

Algorithms, Performance, Theory

\section{Keywords}

Wireless sensor network, self-organization, synchronous cellular automata, asynchronous cellular automata, coverage, connectivity, network lifetime.

\section{INTRODUCTION}

A traditional wireless sensor network (WSN) employs a few numbers of nodes operating as sense and route all the data to the central controller (also known as base station) for further processing. This kind of WSNs relies on the instructions from the central controller or from the system administrator. However, with the changing needs of various applications, the size of WSNs keeps on increasing. Thus, the real time applications may suffer long delays by taking large number of routing hops and the energy holes that will occur in sensor nodes around the central controller because of its acting as a router to pass the information [1]. To resolve the difficulties that centralized control mechanisms face, decentralized algorithms advocate the provision of capabilities to sensor nodes to make decisions and take actions based on the available local information. From the combination of local decisions and actions, global behavior will emerge. System working in this manner can be classified as a self-organizing system [2].

Establishing a self-organized WSN requires a decentralized control mechanism to provide high degree of cooperation among nodes to meet the global goal and switch sensors operating modes to save energy. Cellular automata can be employed for this purpose. CA is a spatially and temporally discrete, deterministic mathematical model and it becomes an important tool to study the space-time evolution of self-organization system due to its capability to reveal the complex system characteristics based on simple local rules. Recently, researchers have attempted to use CA for various self-organized WSNs implementation. Jones et al. $[3,4]$ proposed an energy-efficient data aggregation algorithm for WSN based on CA. Banerjee et al. [5] proposed a sensor node management scheme based on the reversible CA.

The above papers have pointed out that the sensor nodes can be designed to act autonomously to cooperate with neighboring sensor nodes to meet the needs of the applications. In this paper, we investigate how different types of interactions among the nodes affect the system behavior and performance. We quantitatively analyze many types of interactions and discuss the benefits and issues of using CA based algorithm to construct a self-organized WSN.

The rest of this paper is organized as follows. In section 2, we overview cellular automata and a popular CA application, game of life. Section 3 presents the proposed CA based WSN in detail and provides the formulas used for quantitative analysis. Section 4 describes how the simulation is built and the results are discussed. Finally, section 5 provides conclusions and plans for future work. 


\section{CELLULAR AUTOMATA}

CA was proposed by von Neumann in the early 1950s to act as a simple model of biological self-reproduction. Many variants of CAs have been introduced over the years, but most of them have several generic characterizes. In general, they contain a one-, twoor three dimensional lattice of cells and all the cells are equivalent. At time $t$, a cell stays in one of a finite number of possible discrete states. By interacting with the neighbors, a cell updates its current state following a specific transition rule.

According to the above description, a CA can be mathematically defined as a tuple (L, S, N, f) where:

$\mathbf{z} \quad$ L denotes the type of lattices, including dimensions and shape. The elements of lattices are known as cells,

$\mathbf{z} \quad \mathbf{S}$ denotes a finite number of discrete states that a cell can take,

$\mathbf{z} \quad \mathrm{N}$ denotes the number of neighbors that a cell has,

$\mathbf{z}$ f denotes the transition function that a cell follows.

The state transition function of a cell is given as:

$$
S_{t+1}(i)=f\left(\sum_{i=0}^{n} S_{i}(t) \mid i \in(0,1, \mathbf{L}, n)\right)
$$

To explain the CA through a simple example we will use one of the most well-known CA applications which is known as the "Game of Life". This game runs on two-dimensional nonboundary cellular automata. In this system, each cell has one of two possible states in which 1 denotes a cell is alive and 0 for a dead cell. Moreover, each cell updates its state at discrete time instances $t$ simultaneously and follows the below rules:

$\mathbf{z}$ Birth: updates its state from 0 to 1 when it has three live neighbors.

$\mathbf{z} \quad$ Death: updates its state from 1 to 0 when (1) the living cell has no more than one live neighbor or (2) the living cell has more than three neighbors.

$\mathbf{z}$ Survival: maintains the current state when it has two or three live neighbors.

The above rules can be described mathematically by the following formulas:

Birth/Death Rule:

$$
S_{t+1}(i, j)=\left\{\begin{array}{l}
1, \sum_{i=0, j=0}^{n, m} S_{i}(t)=3 \mid \operatorname{exclude}(\mathrm{i}, \mathrm{j}), \\
0, \sum_{i=0, j=0}^{n, m} S_{i}(t) \neq 3 \mid \operatorname{exclude}(\mathrm{i}, \mathrm{j}),
\end{array}\right.
$$

Survival/Death Rule:

$$
S_{t+1}(i, j)=\left\{\begin{array}{l}
1, \sum_{i=0, j=0}^{n, m} S_{i}(t)=2,3 \mid \operatorname{exclude}(\mathrm{i}, \mathrm{j}), \\
0, \sum_{i=0, j=0}^{n, m} S_{i}(t) \neq 2,3 \mid \operatorname{exclude}(\mathrm{i}, \mathrm{j}) .
\end{array}\right.
$$

Unlike one-dimensional CA where the transition rule can be easily described by a rule number explicitly (e.g. rule 90), the transition rule for two-dimensional CA is not convenient and cannot be expressed by the same method. In order to express the rule easily, we depict the rules for Birth/Survival in the same way used in the "Game of Life". Therefore, the above transition rules can be denoted as B3/S23. In this case, B3 and S23 represent equations 2.2 and 2.3 , respectively.

\section{CELLULAR AUTOMATA BASED WSN}

To model the WSN using cellular automata, we make the

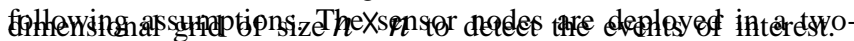
The WSN is running under flat structure (no cluster head exists) and all the nodes have the same properties. Furthermore, no two nodes are located in the same position, which means the maximum number of nodes can be deployed in the grid is $n^{2}$.

Each sensor is considered as a cell in CA and it has three states, active, sleep and dead. In the active mode, the sensor would be performing certain tasks while monitoring an area equivalent to Moore neighbors (8 neighbors) (i.e. it only communicates with

these neighbors). In the sleep mode, the sensor node is saving energy due to its duty has completely performed by other nodes. In the dead mode, the sensor node runs out of energy and it is no longer able to perform any task or cooperates with its neighbors. We consider the active mode of sensor node as the active state of a cell in CA, the sleep mode and the dead mode of sensor node as the death state of a cell in CA. In addition, the sensor node in our model has other properties than the CA cell, namely, residual energy, internal clock and position. Battery provides the power to sensor node, the residual energy is used to indicate the battery status and measure how much longer the sensor node can be last. If a sensor runs out energy, it can not participate in forthcoming actions. The internal clock is used to count when the sensor starts to perform the tasks and change its state based on the local rules at the appropriate time. The position provides the location information to sensors to discover their neighbors. In this paper, a two-dimensional coordinate $(\mathrm{x}, \mathrm{y})$ is used to describe the location.

In order to better depict the system behavior and measure whether the system achieves the design goal, we adopt two important metrics, coverage and connectivity. Coverage is the primary function of a WSN, it represents the capability of WSN to monitor a given area. If the given area is fully covered by at least 1 sensor, this area is considered as 1-coverage. If the given area is covered by at least k sensors, this area is considered as k-coverage [6]. We assume that if any one of 9 cells is alive, the 9 cells area is fully covered by it. Therefore, in our model, the k-coverage criterion is converted to a given $3 \times 3$ grid, at least $k(k \in[1,9])$ sensor(s) is(are) in the active mode. To mathematically determine whether k-coverage can be met in such area, a [0,1] checking function $\Phi$ is given below:

$$
\Phi\left(\sum_{i=1}^{i+1} \sum_{j-1}^{j+1} S_{t}(i, j)\right) \geq k=\left\{\begin{array}{l}
1, k \in[1,9] \\
0, \text { otherwise }
\end{array}\right.
$$

where $(i, j)$ is the position of the central cell for a given $3 \times 3$ grid; and the system coverage is defined as the sum of the checking function for all $3 \times 3$ grids in the system. For a given 
system which contains $n \times n$ cells, only the cells located at the ragged edge do not have 8 neighbors, the remaining cells $(n-2)^{2}$ are taken into account. The formula to measure the system coverage at time $t$ is given below:

$$
\operatorname{Cov}_{\text {sys }}(t)=\frac{\sum_{i=1}^{n-2} \sum_{j=1}^{n-2}\left(\Phi\left(\sum_{i-1}^{i+1} \sum_{j-1}^{j+1} S_{t}(i, j)\right)\right)}{(n-2)^{2}}
$$

Connectivity is another important metric because data from each sensor need to travel multiple hops to reach the destination (e.g. base station). To satisfy this requirement, within the sensor communication range, at least another working sensor exists is the essential condition. Zhang and Hou [8] have proved that if a sensors network provides complete sensing coverage, it can also provides full connectivity when it satisfies the condition that the radio transmission range is at least twice the sensing range. This result indicates that if the sensors can be configured to meet this condition then guarantees for system coverage are transformed to the guarantees of system connectivity. However, in the CA based model, the above condition is not satisfied all the time. In CA, the interaction range of a cell is always restricted to its instant neighbors. For example, the Moore neighbor, a cell is only able to communicate the cells next to it, which means the communication range equals 1 . And the sensing range in our model at least equals 1 (the central cell equals 1 and the other cells are greater than 1 ). Therefore, to evaluate the system connectivity, we can not simply follow the existing approach to convert the connectivity problem to coverage problem, thus another measurement needs to be defined. In CA, the connectivity of a cell can be considered as a living cell has at least one living neighbor. The checking function is given below:

$$
\left.\Phi\left(\sum_{i=1}^{i+1} \sum_{j-1}^{j+1} S_{t}(i, j)\right) \geq k \mid S_{t}(i, j)=1\right)=\left\{\begin{array}{l}
1, k=[2,9] \\
0, \text { otherwise }
\end{array}\right.
$$

where $k$ is the number of live cells in the concerned area and it can be varied to meet the users' specific application needs. The system connectivity can be defined as the percentages of live cells have live neighbors. Unlike system coverage, all the cells in the system are taken into account. The formula at particular time $t$ can be described by the following,

$$
\operatorname{Conn}_{\text {sys }}(t)=\frac{\left.\sum_{i=0}^{n-1} \sum_{j=0}^{n-1}\left(\sum_{i=1}^{i+1} \sum_{j-1}^{j+1} S_{t}(i, j)\right) \geq k \mid S_{t}(i, j)=1\right)}{\sum_{i=0}^{n-1} \sum_{j=0}^{n-1} S_{t}(i, j)}
$$

\section{SIMULATION}

To investigate the system behaviors of CA based WSN, a java simulator has been implemented. The simulator adopts part of the parameters and metrics used in [7]. In the simulator, the sensors are deployed into a $30 \times 30$ regular grid. Each sensor contains $1 \mathrm{~J}$ total energy and it cannot be recharged in the middle of its operation. When the sensor is in active mode, it consumes $0.0165 \mathrm{~J}$ per time unit while in the sleep mode; it consumes
0.00006J per time unit [7]. When the simulation begins, all the sensors choose one of two possible states with identical probability and the simulation will not be terminated until all the sensors run out of energy.

Apart from the coverage and connectivity, several other traditional metrics for wireless sensor networks are also used to assist in analyzing the system behaviors and performance which are:

1. Network lifetime: the duration of time when any sensor in the system is alive.

2. Live nodes: the total number of sensors that still have energy.

3. Active nodes: the total number of sensors that stay in the active mode at a particular time $t$.

4. Global energy: the sum of the residual energy remaining on all nodes at a particular time $t$.

By taking applying different kinds of rules, complex spatialtemporal characteristics emerge from the operations of the CA based WSN. According to a previous CA study [9], regardless of what initial states and evolution rules are used in CA, the system, finally, will fall into one of four basic qualitative behavioral classes:

Class1: All cells eventually attain the same value.

Class2: Simple stable states or periodic and separated structure emerge.

Class3: Chaotic non-periodic patterns are generated.

Class4: Complex, localized, propagating structures are formed.

Certainly, the CA based WSN will exhibit one of the above four behaviors during its lifetime, however, whether the system can still achieve the prospective design goals, e.g. coverage and connectivity, need to be further studied. Through running various types of local rules by varying the values in the birth/survival rules, some interesting regular patterns have emerged from the system. These patterns can be classified into three classes, attenuation, periodic oscillation and homeostasis. In the following, we briefly discuss these patterns.

\subsection{Attenuation}

In this mode, the coverage and the connectivity of system drop to 0 via few steps of interactions based on the local rules from B3/S3 to $\mathrm{B} 8 / \mathrm{S} 8$. In the following, we give an example to demonstrate this situation using local rule B3/S3 which B3 means if a sensor is in the sleep mode, it detects 3 neighbors around it are in the active mode, it turns itself to the active mode and S3 means if a sensor stays in the active mode, it detects 3 of its neighbors are in the active mode, it remains in its current state. The system coverage and connectivity are shown in the Figure 1.

This kind of system can be considered as one that evolves to a fixed point which is attracted to a final state and no longer leaving this state during its remaining lifetime. It is corresponds to the Class1 CA. In our experiments, each sensor contains enough energy to perform further tasks, but the local rule leads to a dysfunctional system. This mode is meaningless for designing a self-organized WSN; and it should be avoided. 

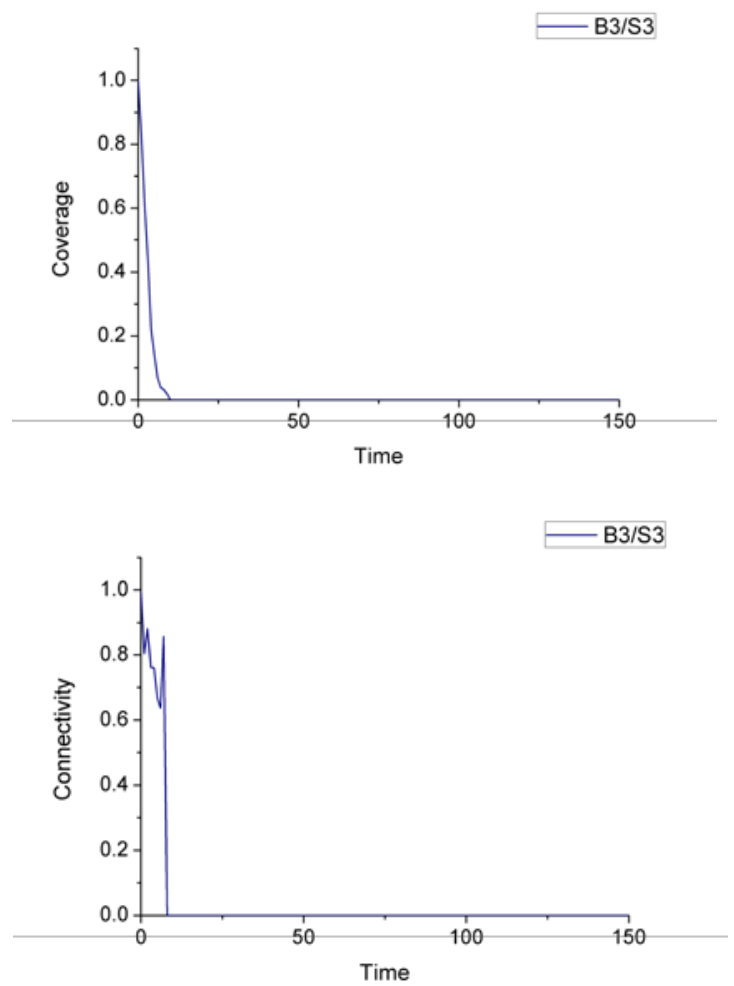

Figure 1. The coverage and the connectivity of the CA based system under rule $\mathrm{B} 3 / \mathrm{S3}$.

\subsection{Periodic oscillation}

In this mode, at most of time, the system exhibits the $0-1$ periodic oscillation behavior both on the network coverage and connectivity based on local rules like B01/S01 and B0/S0. It is corresponds to class2 CA and the system coverage and connectivity are shown in the Figure 2. This indicates that the WSN becomes a periodic functional system, which in most designs it should be avoided. However, if the detected events occur deterministically and periodically, this pattern can be used to wake up the system at the appropriate time to perform tasks in an attempt to save energy and prolong network lifetime due to the fact that for half of the lifetime half of the sensors are in sleep mode.

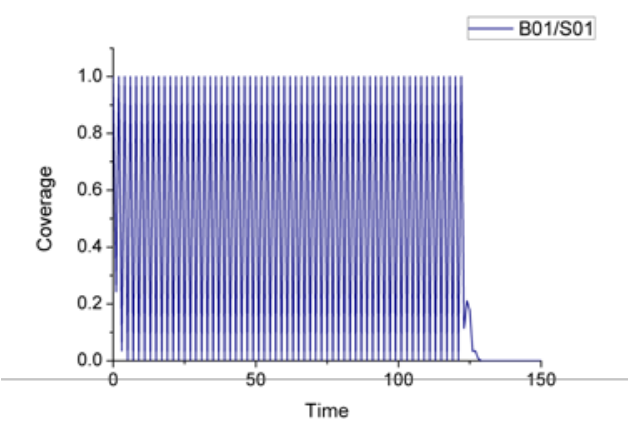

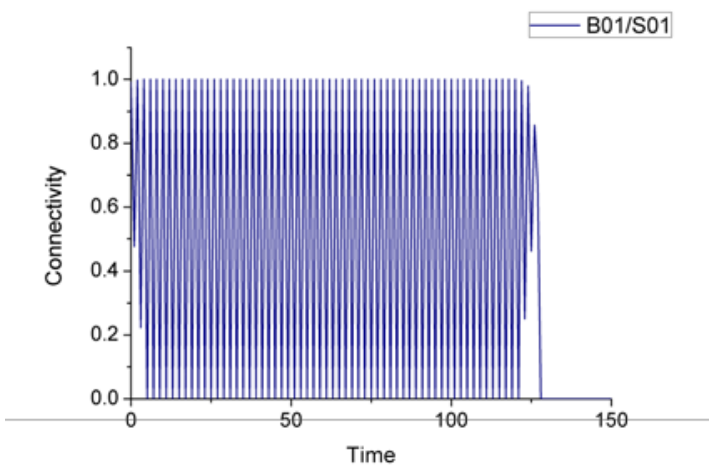

Figure 2. The coverage and the connectivity of the CA based system under rule B01/S01.

\subsection{Homeostasis}

In this mode, through a short period vibration, the system coverage and connectivity converge to a stable non-zero value under the effect of certain local rules (e.g. B1/S01, B34/S34) and stay in this state as long as possible. This pattern can not be strictly mapped to class3 or class4 CA. As observed from Figure 3, through a few steps of internal interactions, the system converges to a steady state, after that, any perturbation (internal or external) tends to make it leave that state, however, the system will automatically eliminate the impact and returns to the steady state. For the CA based WSNs, this mode satisfies most design goals and allows the system to mange itself. Therefore, selecting appropriate rules to ensure that the homeostasis mode appears and remains in the system as long as possible should be an important issue for designers.
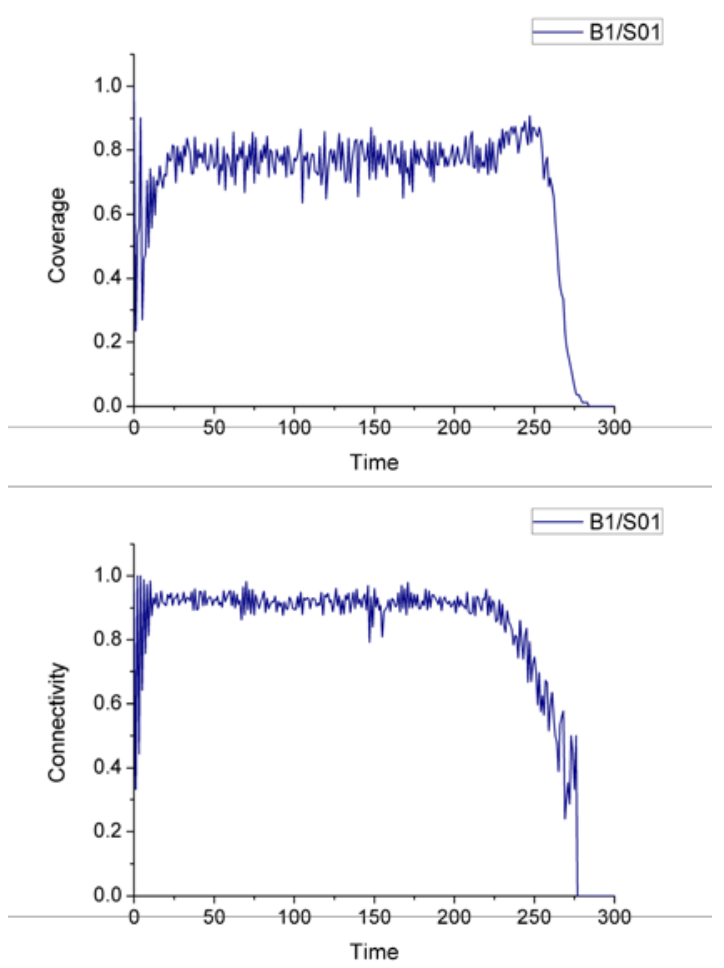

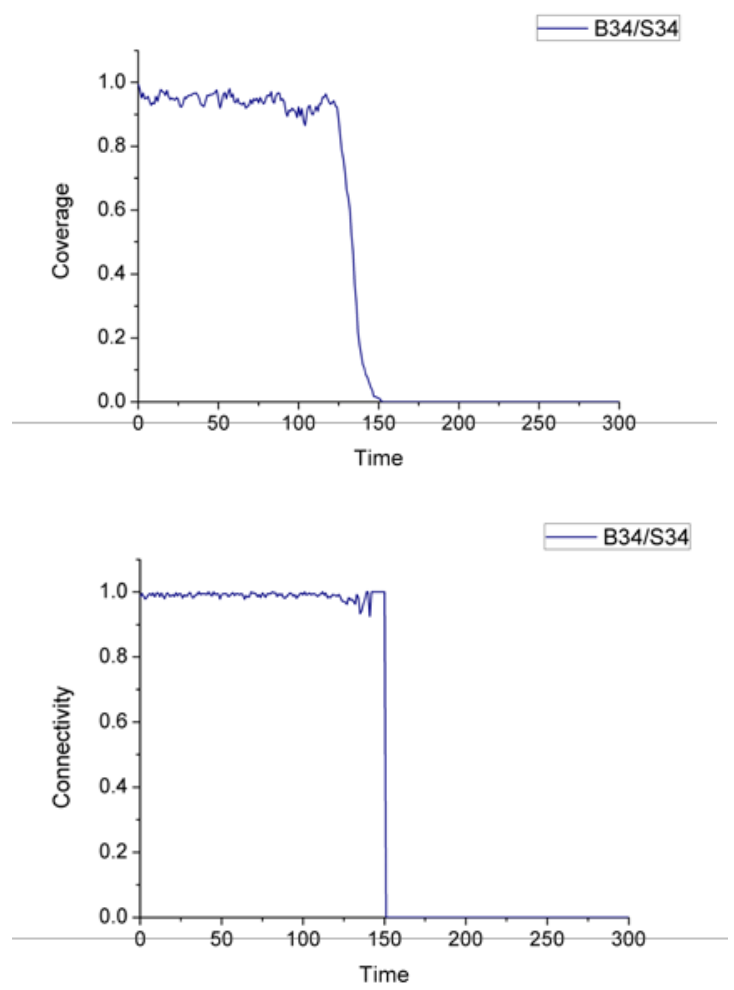

Figure. 3 . The coverage and the connectivity of the CA based system under rule B1/S01 and B34/S34, respectively.

As shown in the Figure 3, by using different local rules from the set of homeostasis mode, the system demonstrates similar behaviour. However, these rules have resulted in different network lifetime. For example, the system that adopted the B34/S34 local rule consumes more energy than the one that adopted B1/S01. So, in order to investigate the system energy consumption when it achieves the same design goals under different conditions, we define a function to evaluate the system energy efficiency issue. The formula is as follows:

$$
\text { eff } f_{\text {sys }}(t)=\frac{\text { ActiveNodes }}{\text { AliveNodes }}
$$

The larger the value of $e f f_{\text {sys }}$ the more energy the system needs to consume to achieve the design goal and this leads to shorter life span. By using equation (4.1), the energy efficiency of the systems with rule B1/S01 and B34/S34 is approximately 0.23 and 0.42 , respectively. Although the system with rule B34/S34 provides better performance on coverage and connectivity than the B1/S01, it pays more cost for achieving this.

Without considering other factors, rule B1/S01 is better than B34/S34 for the CA based WSN to achieve longer network life span. From the system point of view, it can be noticed that at a particular time $t$, not all live sensor nodes are involved in the production of the overall system behaviour, only the active nodes dedicates their effort and form the system to perform the tasks. So, to evaluate how important is a sensor for the system at time $t$, we define the contribution rate of each sensor to measure this issue. For example, we want to measure the contribution rate for a sensor to construct a self-organized system. Based on the information theory, the order of a self-organization system can be described by entropy. The entropy is defined as:

$$
H(X)=-\sum_{i=1}^{n} p\left(x_{i}\right) \log p\left(x_{i}\right)
$$

where $n$ is the total number of active nodes, $p\left(x_{i}\right)$ is the probability of a sensor node $i$ selecting to perform this task. Therefore, the contribution rate for a sensor node for a self-organization WSN is:

$$
R(t)=\frac{H(X)}{\text { ActiveNodes }}
$$

With a less number of active nodes a sensor will have a larger average contribution. This means that each active node in this case has high importance. However, the large value also indicates the system relies more on those sensors. This will decrease the reliability and flexibility of the system, and more importantly, it reduces the possible paths for system evolution. Thus, the balance of between system self-organization and energy efficiency should be seen as a trade-off issue.

In the above, all the CA based WSNs use a synchronous update mechanism. However, in our study, we found that it is not possible to achieve homeostasis when the system starts with the same state (all 0 or all 1 ) regardless of what rule is used. In addition, to implement synchronous a CA based WSN we need to deploy a time synchronization algorithm on each sensor node to ensure they update the states at the same time. This will require extra computational resources and communication bandwidth drawn from limited resources.

\subsection{Asynchronous CA based WSN}

To overcome the abovementioned issues, asynchronous CA is employed in our study. An asynchronous CA is capable to update individual cells independently in such a way that the new state of a cell affects the calculation of states in neighbouring cells. In our simulation, we tend to reach the maximum network lifetime by activating minimum number of sensors and ensure the system behaviour of 1-coverage and 1-connectivity. To meet these requirements the local rule B01/S01 is used. We selectively adopt the values used in the [7] where each sensor checks the states of its immediate neighbours at any time between 1 to 5 time units. Unlike the algorithm in [7] at any particular time in our system only one sensor checks its neighbours' state and updates its own based on the B01/S01 rule. This update method ensures no two cells in the same area will be involved in the state renewal, and it also helps to avoid the deadlock and other unexpected situations that might happen. The result of coverage and connectivity are shown in Figure 4.

It can be noticed from Figure 4 that the system's behaviour remains stable at most of time. The system coverage is above $95 \%$ of the area of interest and it is a satisfactory result for real world applications. In addition, the system connectivity is around $60 \%$ when system coverage reaches $100 \%$. The system only turns unstable and loses function significantly close to the network's death. As discussed earlier, the rule B01/S01 in the synchronous CA based WSN will lead the system to enter into a periodic oscillation mode. But here, the system reaches homeostasis by using the same rule. So this shows that a self-organizing WSN is not affected by the local rule but also by the way different nodes 
interact. In addition, asynchronous CA based WSN has a longer lifetime than a synchronous CA based system in this case.
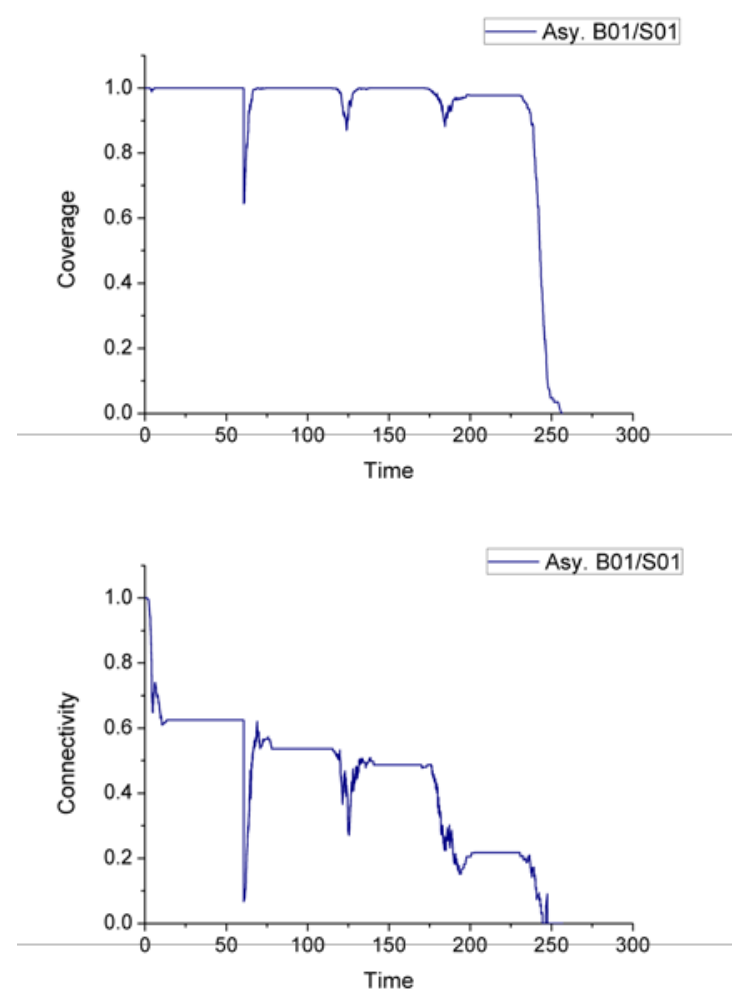

Figure 4. The coverage and the connectivity of the asynchronous CA based WSN under rule B01/S01.

\section{CONCLUSION AND FUTURE WORK}

This paper investigated a CA based WSN at a number of levels. Several formulas are given to describe the system behavior. For a synchronous CA based WSNs some useful patterns are observed and suggestions are provided to design them. To resolve the difficulties associated with synchronous CA based WSNs an asynchronous CA is introduced and different system behaviors are also analyzed.

The sensor is simply mapped to a cell in the current study. A more complex and sophisticated sensor model will need to be developed to better characterize CA based WSNs in the future. The energy is no longer considered as the only resource that will be consumed when the sensor performs a task, the computation capability and communication bandwidth will also need to be considered. In addition, all the sensors are homogeneous for a given task which makes the contribution ratio of each sensor to the self-organized system the same. However, in real world applications, a sensor network may be running multiple applications simultaneously. Each sensor plays a different role in the different applications and the contribution ratio to the system should be varying by taking different roles at different time. By working out the distribution of the contribution rate will enable us to calculate the self-organization degree in the future.

\section{REFERENCES}

[1] S. Olariu and I. Stojmenovic, "Design Guidelines for Maximizing Lifetime and Avoiding Energy Holes in Sensor Networks with Uniform Distribution and Uniform Reporting," in the Proceedings of the $25^{\text {th }}$ IEEE International Conference on Computer Communications (INFOCOM), pp. 1-12, 2006.

[2] H. Haken, Information and Self-Organization: A Macroscopic Approach to Complex Systems: Springer Verlag, 2006.

[3] K. H. Jones, K. N. Lodding, S. Olariu, L. Wilson, and C. Xin, "Biology-Inspired Distributed Consensus in MassivelyDeployed Sensor Networks," Lecture Notes in Computer Science, vol. 3738, p. 99, 2005.

[4] K. H. Jones, K. N. Lodding, S. Olariu, L. Wilson, and C. Xin, "Energy Usage in Biomimetic Models for MassivelyDeployed Sensor Networks," Lecture Notes in Computer Science, vol. 3759, p. 434, 2005.

[5] I. Banerjee, S. Das, H. Rahaman, and B. K. Sikdar, “CA Based Sensor Node Management Scheme: An Energy Efficient Approach,” in the Proceedings of the International Conference on Wireless Communications, Networking and Mobile Computing (WiCom), pp. 2795-2798, 2007.

[6] Y. Fan, G. Zhong, J. Cheng, L. Songwu, and Z. Lixia, "PEAS: A Robust Energy Conserving Protocol for LongLived Sensor Networks," in the Proceedings of the $23^{\text {rd }}$ International Conference on Distributed Computing Systems, pp. 28-37, 2003.

[7] R. O. Cunha, A. P. Silva, A. A. F. Loreiro, and L. B. Ruiz, "Simulating Large Wireless Sensor Networks Using Cellular Automata," in $38^{\text {th }}$ Annual Simulation Symposium, pp. 323330, 2005.

[8] H. Zhang and J. C. Hou, "Maintaining Sensing Coverage and Connectivity in Large Sensor Networks," Ad Hoc\&Sensor Wireless Networks, vol. 1, pp. 89-124, 2005.

[9] N. H. Packard and S. Wolfram, “Two-Dimensional Cellular Automata,” Journal of Statistical Physics, vol. 38, pp. 901946, 1985. 